\title{
Leader sequences are not signal peptides
}

\section{To the editor:}

An article in the June issue (Nat. Biotechnol. 22,773-774, 2004) highlights the challenge of producing a shared vocabulary that biologists can use to communicate their results effectively, explore data and extend scientific investigations. The success of 'bio-ontologies' will be dependent on the use of clearly defined scientific terms. In this context, we feel that ambiguities in the usage of the terms 'leader sequences' and 'signal peptides' require clarification.

The translocation of secretory proteins across intracellular membranes and final localization are mediated by 'address tags' contained within their amino acid sequences. Signal peptides, comprising the N-terminal 13-36 amino acids of secretory proteins, are necessary for the translocation across the first membrane on the secretory pathway and thus universally control the entry of all proteins to the secretory pathway in eukaryotes and prokaryotes. Leader sequences are polynucleotide regions located between the promoter and the coding region and are involved in the regulation of gene expression. Part of the leader sequence may be translated into a short leader peptide but, in contrast to signal peptides, leader peptides are at no time part of the structural proteins. However, although signal peptides and leader sequences clearly represent different entities and have different functions they are often used synonymously to depict signal peptides.

Although the double use of leader sequences to also depict signal peptides is accepted by the International Union of Pure and Applied Chemistry ${ }^{1}$ and the DNA Database of Japan (DDBJ)/European Molecular Biology Laboratory (EMBL, Heidelberg, Germany)/GenBank (http://www.ebi.ac.uk/embl/Documentation/ FT_definitions/feature_table.html), we think the openness of the concept depending on the context is misleading and neglects the significance of leader sequences. A clear definition of the leader sequence, such as the one provided by the Medical Dictionary Search Engine (http://www.books.md/index.html) is strongly preferred. To clarify the difference, we briefly summarize below the general principles of signal peptides and leader sequences.

In eukaryotes, the signal peptide of a nascent precursor protein (pre-protein) directs the ribosome to the rough endoplasmic reticulum (ER) membrane and initiates the transport of the growing peptide chain across $\mathrm{it}^{2,3}$. In prokaryotes, the signal peptide directs the pre-protein to the cytoplasmic membrane. However, the signal peptide is not responsible for the final destination of the mature protein; secretory proteins devoid of further address tags in their sequence are by default secreted to the external environment.

Although signal peptides are not highly conserved, they have a common positively charged n-region, a hydrophobic h-region and a neutral, polar c-region (Fig. 1a).

The c-region contains a weakly conserved cleavage site recognized by membrane-bound signal peptidases. Before the translocation of the pre-protein across the ER membrane, a ribonucleoprotein called signal recognition particle (SRP) binds to the signal peptide emerging from the ribosome. Then the SRP-signal peptide-ribosome complex binds to the ER membrane via an SRP receptor. The signal peptide is then inserted into the ER membrane via a signal peptide binding protein and the nascent polypeptide then crosses the ER membrane through a transmembrane channel. In prokaryotes, pre-proteins are translocated through the cytoplasmic membrane via a similar mechanism. During the translocation across the ER membrane in eukaryotes and the cytoplasmic membrane in prokaryotes, the signal peptide is normally cleaved off the preprotein by a signal peptidase residing in the ER or cytoplasmic membrane.

Leader sequences comprise a short open reading frame coding for a leader peptide and a downstream adjacent region with the propensity of forming mutually exclusive secondary structures (stem-loops) by basepairing of complementary sequences. The formation of one or the other possible stemloops depends on stalling of the ribosome during translation of the leader peptide, either because of lack of the necessary tRNA or because of binding of a specific metabolite to the ribosome/mRNA complex. In turn, the formation of the alternative mRNA conformation affects either the continuation of transcription (transcriptional attenuation) $)^{4}$ or the initiation of translation of the protein coding region (translational attenuation) ${ }^{5}$.
Thus, leader sequences may regulate gene expression at the level of transcription or translation. The structure is summarized in Figure $1 \mathbf{b}$.

The type of transcriptional attenuation mechanisms seen in prokaryotic leader sequences also occur in operons encoding enzymes involved in the biosynthesis of other amino acids, antibiotic resistance and glucoside uptake and metabolism. Translational attenuation occurs in antibiotic resistance (e.g., the ermC gene of Staphylococcus aureus ${ }^{5}$ ). In eukaryotes, transcription and translation are physically separated and regulation of gene expression is more complex, involving, for example, mRNA processing. However, leader sequences are also involved in gene expression in eukaryotes and examples of leader sequence-dependent regulation of translation have been described ${ }^{6}$.

\section{Michael Mølhøj ${ }^{1}$ \& Florence Dal Degan ${ }^{2}$ \\ ${ }^{1}$ Micromet AG, Staffelseestrasse 2, D-81477 Munich, Germany and ${ }^{2}$ Pharmexa A/S, Kogle Allé 6, DK-2970 Hørsholm, Denmark. e-mail:fdd@pharmexa.com}

1. International Union of Pure and Applied Chemists. Compendium of Chemical Terminology, 2nd edition. (IUPAC, Research Triangle Park, NC, 1997).

2. Devillers-Thiery, A., Kindt, T., Scheele, G. \& Blobel, G. Proc. Natl. Acad. Sci. USA 72, 5016-5020 (1975).

3. von Heijne, G. J. Membrane Biol. 115, 195-201 (1990).

4. Platt, T. Cel/ 24, 10-23 (1981).

5. Weisblum, B. Antimicrob. Agents Chemother. 39 797-805 (1995).

6. Rook, F. et al. Plant J. 15, 253-263 (1998). 